\title{
A TIME DELAY FOR THE CLUSTER-LENSED QUASAR SDSS J1004+4112
}

\author{
J. Fohlmeister, ${ }^{1}$ C. S. Kochanek, ${ }^{2}$ E. E. Falco, ${ }^{3}$ J. Wambsganss, ${ }^{1}$ N. Morgan, ${ }^{2}$ C. W. Morgan, ${ }^{2,4}$ \\ E. O. Ofek, ${ }^{5}$ D. Maoz, ${ }^{6}$ C. R. Keeton, ${ }^{7}$ J. C. Barentine, ${ }^{8}$ G. Dalton, ${ }^{9}$ J. Dembicky, ${ }^{8}$ \\ W. Ketzeback, ${ }^{8}$ R. McMillan, ${ }^{8}$ and C. S. Peters ${ }^{10}$ \\ Received 2006 July 23; accepted 2007 March 5
}

\begin{abstract}
We present 426 epochs of optical monitoring data spanning 1000 days from 2003 December to 2006 June for the gravitationally lensed quasar SDSS J1004+4112. The time delay between the A and B images is $\Delta t_{\mathrm{BA}}=38.4 \pm$ 2.0 days $\left(\Delta \chi^{2}=4\right)$ in the expected sense that B leads $\mathrm{A}$ and the overall time ordering is C-B-A-D-E. The measured delay invalidates all published models. The models probably failed because they neglected the perturbations from cluster member galaxies. Models including the galaxies can fit the data well, but conclusions about the cluster mass distribution should await the measurement of the longer, and less substructure sensitive, delays of the $\mathrm{C}$ and $\mathrm{D}$ images. For these images, a delay of $\Delta t_{\mathrm{CB}} \simeq 681 \pm 15$ days is plausible but requires confirmation, while delays of $\Delta t_{\mathrm{CB}}>560$ days and $\Delta t_{\mathrm{AD}}>800$ days are required. We clearly detect microlensing of the $\mathrm{A} / \mathrm{B}$ images, with the delay-corrected flux ratios changing from $m_{\mathrm{B}}-m_{\mathrm{A}}=0.44 \pm 0.01 \mathrm{mag}$ in the first season to $0.29 \pm 0.01 \mathrm{mag}$ in the second season and $0.32 \pm 0.01 \mathrm{mag}$ in the third season.
\end{abstract}

Subject headings: cosmology: observations — gravitational lensing — quasars: individual (SDSS J1004+4112)

Online material: machine-readable table

\section{INTRODUCTION}

The wide-separation lensed quasar SDSS J1004+4112 was discovered in the Sloan Digital Sky Survey (SDSS) search for lenses (Inada et al. 2003; Oguri et al. 2004; Sharon et al. 2005; Wambsganss 2003). The lens consists of at least four images of a redshift $z_{s}=1.734$ quasar whose $\sim 15.0^{\prime \prime}$ Einstein ring diameter is created by a redshift $z_{l}=0.68$ cluster. The cluster has been characterized with X-ray observations (Ota et al. 2006; Lamer et al. 2006), and there are additional multiply imaged arcs formed from still higher redshift background galaxies (Sharon et al. 2005). There is also strong evidence for a fifth, lensed image of the quasar located near the center of the brightest cluster galaxy (Inada et al. 2005), which in combination with a future velocity dispersion measurement for the galaxy will strongly constrain the central mass distribution of the lens (e.g., Sand et al. 2004, but see Dalal \& Keeton 2003). Thus, it is not only feasible to cleanly compare X-ray and lensing mass distributions in this galaxy cluster, but it may also be possible to test the cosmological model by measuring the increase of the Einstein radius with source redshift due to the $D_{\mathrm{LS}} / D_{\mathrm{OS}}$ distance ratio scaling of the lens deflection (Soucail et al. 2004).

That the source is a time-variable quasar offers further and unique opportunities for this cluster lens. First, the time delay

\footnotetext{
1 Astronomisches Rechen-Institut, Zentrum für Astronomie der Universität Heidelberg, 69120 Heidelberg, Germany.

2 Department of Astronomy, Ohio State University, Columbus, OH 43210.

3 Smithsonian Astrophysical Observatory, FLWO, Amado, AZ 85645.

4 Department of Physics, United States Naval Academy, Annapolis, MD 21402.

5 California Institute of Technology, Pasadena, CA 91125.

6 School of Physics and Astronomy and the Wise Observatory, Tel-Aviv University, Tel-Aviv 69978, Israel.

7 Department of Physics and Astronomy, Rutgers University, Piscataway, NJ 08854.

8 Apache Point Observatory, Sunspot, NM 88349.

9 Department of Physics, University of Oxford, Oxford OX1 3RH, UK.

10 Department of Physics and Astronomy, Dartmouth College, Hanover, NH $03755-3528$.
}

between the quasar images can be measured as a constraint on the mass distribution. In theory, the time delays determine the mean surface density near the images for which the delay is measured (Kochanek 2002), so the mass sheet $(\kappa)$ degeneracy of most cluster lensing measurements can be broken under the assumption that the Hubble constant is well determined by other means. Several theoretical studies of the time delays in SDSS J1004+4112 (Oguri et al. 2004; Williams \& Saha 2004; Kawano \& Oguri 2006) have explored their dependence on the mean mass profile of the cluster, finding a broad range of potential delays. As we show here, all these models are incorrect in their details because they neglected cluster member galaxies whose deflection scales are larger than the positional constraints on the quasar images used in the models (see the discussion in Keeton et al. [2000] on the failure of similar models for the cluster lens Q0957+561 and the general discussion in Kochanek et al. 2006b). Nonetheless, all these models indicate that the delay between the A and B images is relatively short (weeks) and that its value should indicate the magnitude of the much longer (years) delays of the $\mathrm{C}$ and $\mathrm{D}$ images.

The second unique property of the lens is that microlensing of the quasar accretion disk by any stars in the cluster halo or small satellites near the images can be used as an added probe of the structure of the cluster (see Kochanek et al. 2006b). Because the cluster has a higher velocity dispersion $\left(700 \mathrm{~km} \mathrm{~s}^{-1}\right)$ than a typical galaxy lens $\left(\sim 200 \mathrm{~km} \mathrm{~s}^{-1}\right)$, the microlensing timescales in this system may also be shorter than for a typical lens by about a factor of 3 . There is already evidence for microlensing from the time variability of the $C$ IV $\lambda 1549$ line in image $A$ that is not observed in image B (Richards et al. 2004; Lamer et al. 2006; Gómez-Álvarez et al. 2006), although recently Green (2006) has suggested that this could also be due to time variable absorption in the source quasar.

For 3 years we have conducted an optical monitoring campaign to measure the optical variability of this system. This has proved more challenging than desired because the quasars are somewhat faint for monitoring with available telescopes and 


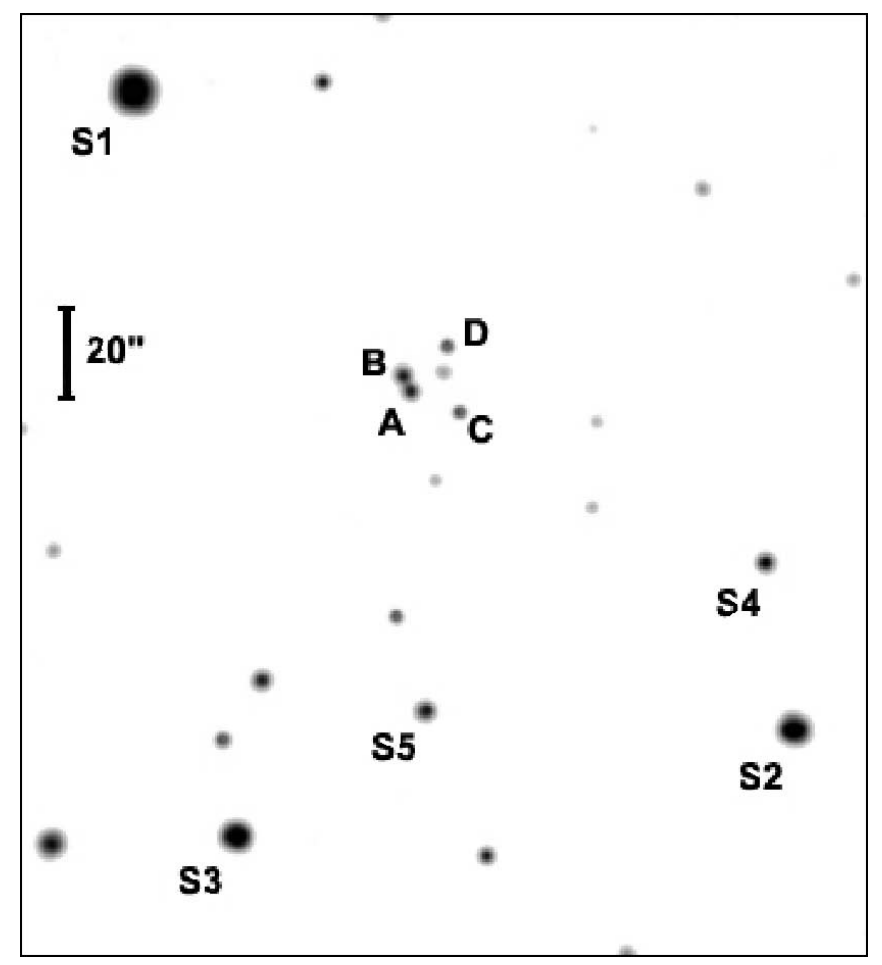

FIG. 1.-The $r$-band image obtained with Minicam on 2005 January 16 . The $3.3^{\prime} \times 3.5^{\prime}$ field shows the lensed images of SDSS J1004+4112 and the five reference stars S1, S2, S3, S4, and S5 used for the PSF.

modest exposure times. However, we have succeeded both in measuring the A/B time delay and clearly detecting microlensing of the optical continuum of the quasar. In $\S 2$ we present the data from the monitoring campaign for the four bright lensed quasar images. In $\S 3$ we determine the A/B time delay, discuss the presence of microlensing in the system, and place constraints on the long delays between the close image pair $\mathrm{A}$ and $\mathrm{B}$ and the fainter images $C$ and D. In $\S 4$ we discuss the failure of existing models for the system and introduce a simple successful model that includes the perturbations of cluster galaxies, and we conclude in $\S 5$.

\section{DATA}

The photometric monitoring observations presented here took place between 2003 December and 2006 June. The bulk of data were taken with the $1.2 \mathrm{~m}$ telescope at Fred Lawrence Whipple Observatory on Mount Hopkins using the 4Shooter $(R$ band, 93 epochs, $0.66^{\prime \prime}$ pixels), Minicam (SDSS $r$ band, 74 epochs, $0.604^{\prime \prime}$ pixels), and Keplercam (SDSS $r$ band, 91 epochs, $0.672^{\prime \prime}$ pixels, plus 4 epochs in $R$ band) during the first, second, and third season, respectively. Additional data were obtained with the Apache Point Observatory (APO) $3.5 \mathrm{~m}$ telescope using SPICam (SDSS $r$ band, 9 epochs, $0.282^{\prime \prime}$ pixels), the MDM $2.4 \mathrm{~m}$ Hiltner

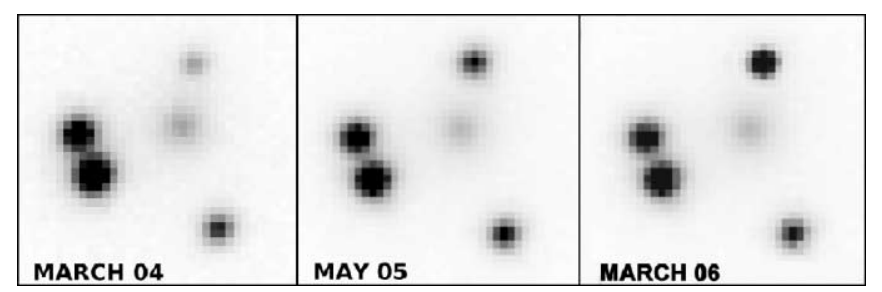

FIG. 2.-The $23^{\prime \prime} \times 23^{\prime \prime}$ insets on the four bright quasar images at three different epochs separated by about $1 \mathrm{yr}$ (for nomenclature see Fig. 1). The faint source in their middle is the bright galaxy belonging to the lensing cluster. telescope using the RETROCAM (Morgan et al. 2005; SDSS $r$ band, 27 epochs, $0.259^{\prime \prime}$ pixels), $8 \mathrm{~K}$ ( $R$ band, 12 epochs, $0.344^{\prime \prime}$ pixels), Templeton ( $R$ band, 8 epochs, $0.275^{\prime \prime}$ pixels) and Echelle ( $R$ band, 3 epochs, $0.275^{\prime \prime}$ pixels) detectors, the MDM $1.3 \mathrm{~m}$ McGraw-Hill telescope using the Templeton detector $(R$ band, 6 epochs, $0.508^{\prime \prime}$ pixels), the Palomar Observatory $1.5 \mathrm{~m}$ telescope using the SITe detector ( $R$ band, 13 epochs, $0.379^{\prime \prime}$ pixels), the Wise Observatory $1.0 \mathrm{~m}$ telescope with the Tektronix ( $R$ band, 30 epochs, $0.696^{\prime \prime}$ pixels) and TAVAS (clear, 53 epochs, $0.991^{\prime \prime}$ pixels) detectors, and the WIYN $3.5 \mathrm{~m}$ telescope using the WTTM (SDSS $r$ band, 3 epochs, $0.216^{\prime \prime}$ pixels) detector. The combined data set consists of 426 epochs.

In Figure 1 the quasar images are labeled A, B, C, and D, following the notation by Inada et al. (2003). The (nonvariable) reference stars used for flux calibration and building the PSF are S1, S2, S3, S4, and S5. The small panels in Figure 2 show snapshots of the four bright quasar images at three different observing epochs, in 2004 March, 2005 May, and 2006 March. These images illustrate how images A and B slowly faded during the course of the three seasons, while image D became significantly brighter. The galaxies of the lensing cluster are not detectable in the individual observations, except for the bright galaxy close to image D (G1 in Oguri et al. 2004). The candidate fifth quasar image, E, lies near the center of this galaxy (Inada et al. 2005).

The data were fitted using the methods of Kochanek et al. (2006a) for HE 0435-1223. Regions around each of the quasar images and the "standard" S1-S5 stars (see Fig. 1) are fitted to determine the relative fluxes and the structure of the PSF. For each filter, the star S1 was defined to have unit flux, while the fluxes of the remaining stars S2, S3, S4, and S5 were adjusted to this calibration standard based on all the available epochs of data for each filter. The relative fluxes of the standard stars depend on the filter, with ratios of 1.0:0.439:0.360:0.130:0.0583 for the $R$ band, 1.0:0.334:0.329:0.0937:0.0613 for the SDSS $r$ band, and $1.0: 0.63: 0.64: 0.39: 0.20$ for the clear filter. In the WIYN/WTTM, MDM $2.4 \mathrm{~m} / 8 \mathrm{~K}$ and MDM $2.4 \mathrm{~m} /$ Templeton data, the star S1 frequently is too close to saturation for use, so its weight in the fits is greatly reduced. It was not necessary to further subdivide the calibrations for the individual detectors given the overall quality of the photometry, as the average calibration offsets between detectors using the same filter were well under $0.01 \mathrm{mag}$. We then matched the $R$-band and clear observations to the $r$-band observations using the quasar light curves themselves. For each $R$ /clear epoch bracketed by $r$-band observations within 1 week, we interpolated the $r$-band observation to the epoch of the other band and computed the mean offset between the light curves. Offsets of $0.043 \pm 0.006$ mag and $0.250 \pm 0.011$ mag must be added to the $R$-band and clear magnitudes, respectively, to match them to the $r$-band data.

We note that our final magnitudes are not fully calibrated to the true $r$ band and that our analysis method derives more from the difference imaging methods used in almost all modern variability studies (Alard \& Lupton 1998) than from classical photometry. In essence, we used the standard stars to put the data for each filter onto a consistent flux scale, and then used the quasars themselves to put the different filters onto a consistent flux scale. Color terms arising from the color differences between the stars and the quasars are unimportant because our analysis and results depend only on flux ratios between the quasars. The inclusion of the clear filter data might be problematic if the quasar images had very different colors. Empirically, eliminating the clear filter data has no effect on our results.

Figure 3 shows the resulting light curves for images A-D over the three observing seasons and Table 1 presents the photometry 


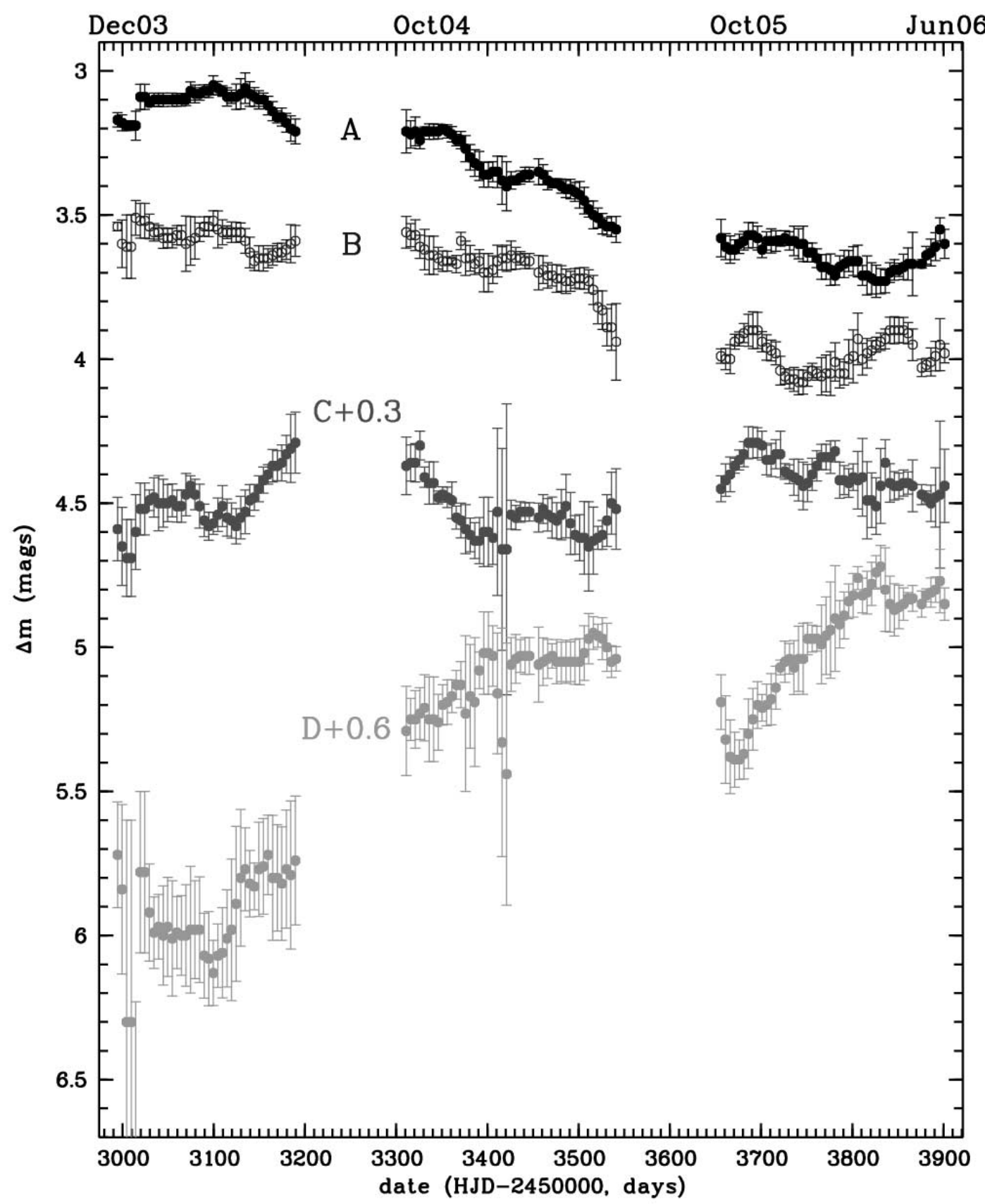

FIG. 3.-Light curves of the A-D images of SDSS J1004+4112 from 2003 December to 2006 June. Here we show a running average of the data (one point every 5 days averaged over \pm 7 days) to emphasize the trends rather than the noise. Images $C$ and $D$ have been offset by 0.3 and 0.6 mag because they would otherwise overlap with each other and with image $B$ in the third season.

for the four images. They span a time period of 1000 days from 2003 December to 2006 June with two seasonal gaps of approximately 100 days during the period from July to October. SDSS J1004+4112 is a relatively faint quasar for monitoring with $1 \mathrm{~m}$-class telescopes, and the image quality of the FLWO and WISE telescopes is poor. As a result, the noise in many of the measurements is relatively large compared to the variability amplitude. On the other hand, our sampling cadence is quite high, so the overall statistical power of the data is very good, with a mean sampling rate of once every two days while the source is visible. All four images vary by about $0.5 \mathrm{mag}$, with the more than 1 mag brightening of image $\mathrm{D}$ being the largest change during the three seasons. For the purposes of measuring the A/B time delay, the most interesting features are the minima in the
B light curve near days 3150 and 3750 in the first and third seasons, respectively, and the corresponding features in the A light curve roughly 40 days later. The second season shows no obvious features that can be used to measure the delay. The second important point to note is that the A/B flux ratio has changed significantly between the first and third seasons, indicating that microlensing is occurring in this system as has been previously suggested by variations in the $\mathrm{C}$ IV emission-line profile (Richards et al. 2004; Lamer et al. 2006; Gómez-Álvarez et al. 2006).

\section{THE TIME DELAY}

Model predictions for the time delay of the close image pair A and B are a few weeks (Oguri et al. 2004; Williams \& Saha 2004; Kawano \& Oguri 2006) and therefore should be measurable 
TABLE 1

Light CURVES FOR SDSS J1004+4112

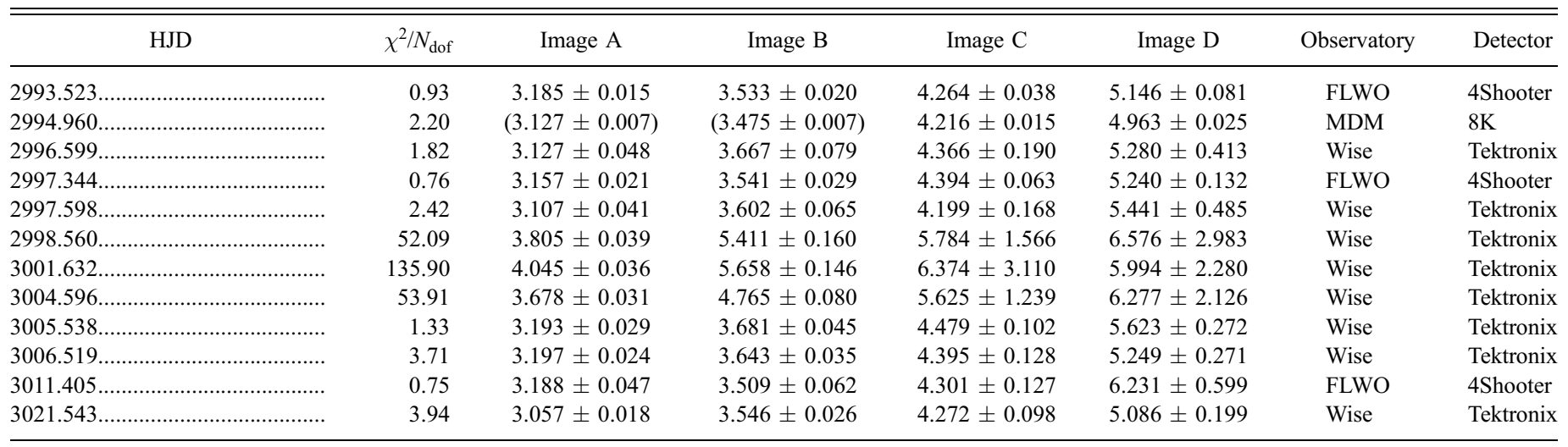

Notes.-Table 1 is published in its entirety in the electronic edition of the Astrophysical Journal. A portion is shown here for guidance regarding its form and content. The heliocentric Julian days (HJD) column gives the date of the observation relative to HJD $=2,450,000$. The $\chi^{2} / N_{\text {dof }}$ column indicates how well our photometric model fit the imaging data. When $\chi^{2}>N_{\text {dof }}$ we rescale the photometric errors presented in this table by $\left(\chi^{2} / N_{\text {dof }}\right)^{1 / 2}$ before carrying out the time delay analysis to reduce the weight of images that were fit poorly. The image magnitudes are relative to the comparison stars (see text). The 16 mag enclosed in parentheses are not used in the time delay estimates.

within each season of the light curves. Of the many techniques for calculating time delays from light curves (e.g., Gil-Merino et al. 2002; Pelt et al. 1994; Press et al. 1992; Kochanek et al. 2006a), we apply three. The three methods produce mutually consistent results, but we adopt the Kochanek et al. (2006a) polynomial method for our standard result because it naturally includes the effects of microlensing on the delay estimate. As is clear from the light curves, image B leads image A, so the delay ordering of the images is C-B-A-D-E. We conclude with a discussion of the longer $\mathrm{C}$ and $\mathrm{D}$ image time delays.

For our analysis of the A/B delay we treated the data in Table 1 as follows. If the goodness of fit of the photometric model to an image had a $\chi^{2}$ statistic larger than the number of degrees of freedom $N_{\text {dof }}$ (see Table 1), we rescaled the photometric errors for that image by $\left(\chi^{2} / N_{\text {dof }}\right)^{1 / 2}$ on the grounds that having $\chi^{2}>$ $N_{\text {dof }}$ meant that the uncertainties were underestimated. For the time delay estimates we dropped the 16 points marked in Table 1 that were more than $3 \sigma$ from the best fitting models. We also repeated the time delay estimates excluding all points with rescaled photometric errors larger than $0.1 \mathrm{mag}$, finding no significant changes.

\subsection{Simple $\chi^{2}$ Minimization}

The simplest approach to the delay measurement problem is to take the observed light curves $\mathrm{A}\left(t_{i}\right)$ and $\mathrm{B}\left(t_{i}\right)$ and cross-correlate them with linearly interpolated light curves $a(t)$ and $b(t)$ for the other image. We assume that the light curves of the two images are the same except for a time delay $\tau$ and a magnitude offset $m(\tau)$. In practice, we use a different magnitude offset for each season to partially compensate for the effects of microlensing. Based on this assumption we can calculate the time delay by minimizing the deviations from $m(\tau)$ for each pair $\left[\mathrm{A}\left(t_{i}\right), b\left(t_{i}-\tau\right)\right]$ and $\left[a\left(t_{i}+\tau\right), \mathrm{B}\left(t_{i}\right)\right]$ by a fit statistic

$$
\begin{aligned}
\frac{\chi^{2}(\tau)}{N_{\mathrm{dof}}(\tau)}= & \frac{1}{2 N_{\mathrm{dof}}(\tau)} \sum_{i}^{N(\tau)} \frac{\left[\mathrm{A}\left(t_{i}\right)-b\left(t_{i}-\tau\right)+m(\tau)\right]^{2}}{\sigma_{\mathrm{A}, i}^{2}+\sigma_{b, t}^{2}} \\
& +\frac{1}{2 N_{\mathrm{dof}}(\tau)} \sum_{i}^{N(\tau)} \frac{\left[a\left(t_{i}+\tau\right)-\mathrm{B}\left(t_{i}\right)+m(\tau)\right]^{2}}{\sigma_{a, t}^{2}+\sigma_{\mathrm{B}, i}^{2}}
\end{aligned}
$$

that is symmetric as to which image is being interpolated. The errors in the observed magnitudes are $\sigma_{\mathrm{A}, i}$ and $\sigma_{\mathrm{B}, i}$ and the errors in the interpolated magnitudes are $\sigma_{a, t}$ and $\sigma_{b, t}$. The fit is carried out only where the light curves overlap (i.e., excluding the season gaps), so the number of data points used $N(\tau)$ depends on the delay $\tau$.

Figure 4 shows the results for the three seasons separately and for the combined light curve. Analyzed separately, the first and third seasons show minima at 30 and 46 days, respectively, while there is no clear minimum for the second season due to the lack of significant features in the light curve. For the joint analysis of all three seasons we allowed for an independent value of $m(\tau)$ within each season to model the changes in the flux ratios due to microlensing. The analysis of the combined data yields a delay of $39 \pm 3$ days.

\subsection{The Dispersion Method}

One potential weakness of the simple $\chi^{2}$ method is the need for interpolation. As our second approach we apply the dispersion spectra method developed by Pelt et al. $(1994,1996)$ to

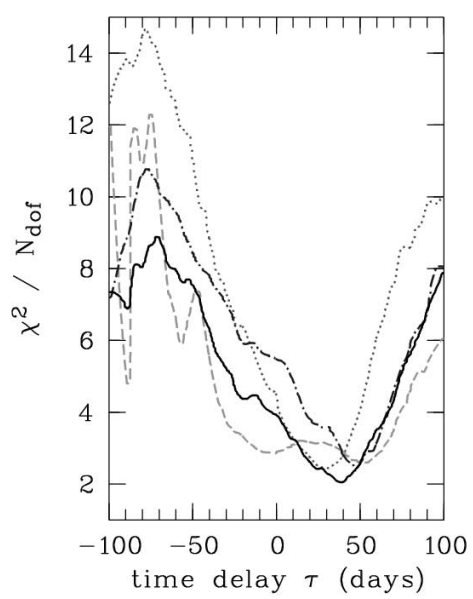

FIg. 4.- Results of the $\chi^{2}$ minimization between the two time series A and $\mathrm{B}$ for the first (dotted line), second (dashed line), third (dash-dotted line), and combined observing seasons (solid line). Note that near the minimum for the combined light curve a change of $\chi^{2} / N_{\text {dof }}$ of 0.01 is statistically significant. 


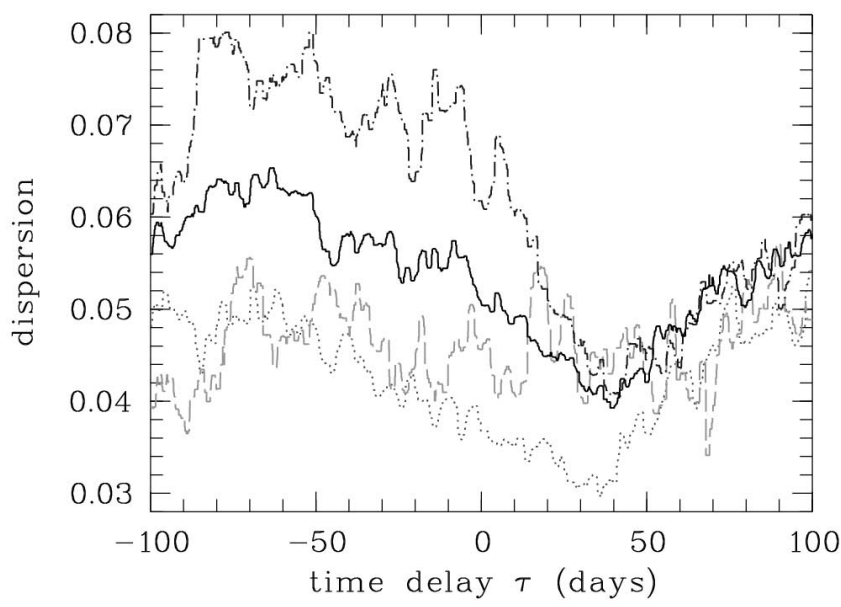

FIG. 5.-Dispersion spectra for the first (dotted line), second (dashed line), third (dash-dotted line), and combined observing seasons (solid line).

avoid the interpolation. Instead, a combined light curve $C(t)$ is constructed by shifting the data points of one image in magnitude $[m(\tau)]$ and time $(\tau)$ and combining them with the data points of the other image

$$
C\left(t_{k}\right)= \begin{cases}\mathrm{A}_{i} & t_{k}=t_{i}, \\ \mathrm{~B}_{j}-m(\tau) & t_{k}=t_{j}+\tau,\end{cases}
$$

where $k=1, \ldots, N$ and $N=N_{\mathrm{A}}+N_{\mathrm{B}}$. The time delay $\tau$ is estimated by minimizing the dispersion spectrum

$$
D^{2}(\tau)=\min _{m(\tau)} \frac{\sum_{k=1}^{N-1} S_{k} W_{k} G_{k}\left(C_{k+1}-C_{k}\right)^{2}}{2 \sum_{k=1}^{N-1} S_{k} W_{k} G_{k}},
$$

where the $W_{k}=\left(\sigma_{k}^{2}+\sigma_{k+1}^{2}\right)^{-1}$ are the statistical weights of the data, $G_{k}=1$ if the points $k$ and $k+1$ come from different images $(\mathrm{A} / \mathrm{B})$ and $G_{k}=0$ otherwise (A/A or B/B), and $S_{k}=1$ if $\left|t_{k+1}-t_{k}\right| \leq \delta$ and $S_{k}=0$ otherwise. We use a decorrelation timescale of $\delta=3$ days, but our results depend little on the exact choice. The results are shown in Figure 5 for both the individual seasons and the combined data. We again used independent estimates of $\Delta m$ for each observing season to compensate for the effects of microlensing. We find 36 and 38 days for the first and third seasons, 38 days for the combined data, and no significant minimum using only the data from the second season.

We estimated the errors using the resampling procedure of Pelt et al. (1994). The combined light curve $C_{k}$ was smoothed for each time delay using a seven-point median filter surrounding each point. Residuals relative to the original data were then reshuffled randomly to create artificially noisy combined light curves. Time delays for a set of 1000 such light curves were determined by calculating the dispersion spectra, leading to the distribution of minimum dispersion estimates shown in Figure 6. If we define the uncertainties by the range about the median encompassing $68 \%$ of the random trials, we estimate that the uncertainty in the time delay is \pm 6 days.

\subsection{The Polynomial Method}

The clear indication of microlensing effects means that corrections for microlensing are required to determine an accurate time delay. Both the $\chi^{2}$ and minimum dispersion methods treated

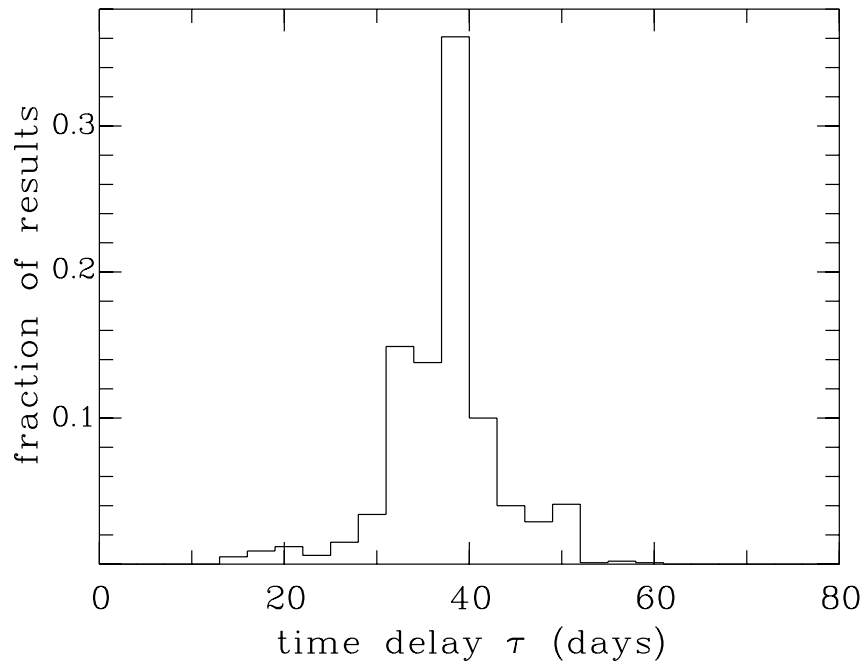

FIG. 6.- Results of the resampling procedure in the dispersion method. From the width of the distribution we estimate the uncertainty for the time delay measurement in the dispersion spectra.

the flux ratios between the images within each season as a constant. Either method could be modified to allow for more complex microlensing variations, but for our final analysis we will use the polynomial fitting method of Kochanek et al. (2006), since it can most easily incorporate the effects of microlensing on both the delays and their uncertainties.

In the Kochanek et al. (2006) polynomial method, the time variations of the source are modeled as a Legendre polynomial of order $N_{\text {src }}$, and the time variations due to microlensing are modeled as a Legendre polynomial of order $N_{\mu}$ in each of the three seasons. The amplitudes of the coefficients of the source polynomial are weakly constrained to match the structure function measured for SDSS quasars by Vanden Berk et al. (2004). The polynomial orders are determined by using the $F$-test to indicate which polynomial order no longer leads to statistically significant improvements in the fits. We used polynomial orders of $N_{\mathrm{src}}=20,40$, and 60 and $N_{\mu}=0,1$, and 2 . The microlensing polynomial orders correspond to using a constant flux ratio, a linear trend or a quadratic trend for each season. Based on the $F$-test, the improvement in the fit to the data is significant when jumping from $N_{\mathrm{src}}=20$ to 40 and from $N_{\mu}=0$ to 1 (from constant flux ratios in each season to linear trends), but not for any of the higher order models. The delays for all the cases are consistent with each other given their uncertainties, so we will adopt the result for the $N_{\mathrm{src}}=60, N_{\mu}=3$ model, $\Delta t_{\mathrm{BA}}=38.4 \pm$ 1.0 days $\left(\Delta \chi^{2}=1, \pm 2.0\right.$ days at $\Delta \chi^{2}=4$, see Fig. 7). Using higher than necessary polynomial orders should be conservative and overestimate the uncertainties in the time delay. The overall fit has $\chi^{2}=718$ for $N_{\text {dof }}=663$.

In this model, the mean magnitude differences between $\mathrm{A}$ and $\mathrm{B}$ for the three seasons are $0.439 \pm 0.008,0.292 \pm 0.012$ and $0.321 \pm 0.008 \mathrm{mag}$, with seasonal gradients of $-0.10 \pm 0.03$,

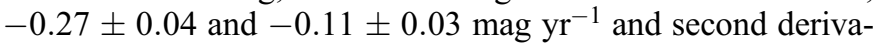
tives of $-1.2 \pm 0.4,0.6 \pm 0.5$ and $-0.7 \pm 0.3 \mathrm{mag} \mathrm{yr}^{-2}$, respectively. Thus, microlensing is clearly present, as expected from the visible structure of the A and B light curves. The need to model the microlensing as more than a seasonal change in the flux ratio means that the polynomial models fit the data considerably better than the first two methods, which is one reason for the significantly smaller formal uncertainties in the delay. Using only the higher precision data points has a negligible effect on the delays or the inferred level of microlensing. Figure 8 shows the 


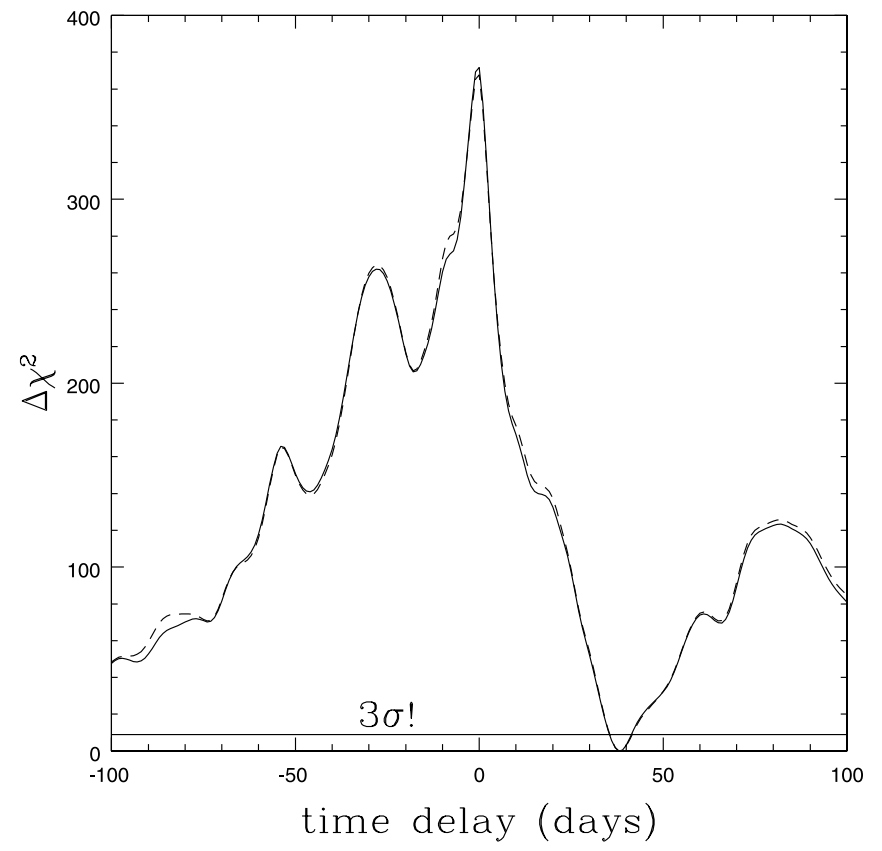

Fig. 7.-Polynomial method $\chi^{2}-\chi_{\min }^{2}$ for the standard model we adopt for the delay. The dashed line uses all data, and the solid line uses only data with uncertainties under $0.1 \mathrm{mag}$. The horizontal line shows the $\Delta \chi^{2}=9$ level corresponding to a $3 \sigma$ change in $\chi^{2}$. The dropping $\chi^{2}$ at the edges is due to the diminishing overlap of the light curves, which allows the source variability to start fitting the two separate light curves independently and "perfectly."

estimated source light curve as compared to the data, and Figure 9 shows the inferred level of microlensing variability. We can only measure the differential microlensing between $\mathrm{A}$ and $\mathrm{B}$, and the choice of assigning it to image B is an arbitrary one which does not affect the time delay estimate.

\subsection{Constraints on the Long Delays}

The model predictions for the long time delays between the close image pair A and B and the fainter images $C$ and D are very uncertain. For example, Oguri et al. (2004) found an approximate scaling relation of $\Delta t_{\mathrm{CD}} / \Delta t_{\mathrm{BA}}=143 \pm 16$ for their models, which would imply a $15 \mathrm{yr} \mathrm{C} / \mathrm{D}$ time delay given our results for the A/B time delay. On the other hand, Williams \& Saha (2004) found delay estimates of order $\Delta t_{\mathrm{CB}} \approx 400$ days and $\Delta t_{\mathrm{AD}} \approx$ 600 days, albeit with a large scatter (about \pm 200 days). As we discuss in $\S 4$, these studies use simplified mass models that are of only limited use for calibrating our expectations.

Empirically, with our 1000 day time span for the light curves we can test for image $\mathrm{C}$ and $\mathrm{D}$ delays of \pm 1000 days. We did so by matching the $\mathrm{C}$ or $\mathrm{D}$ light curve to the combined A/B light curves using the polynomial method. Since the overall behavior of the A and B light curves during the first and second season is mainly decreasing or flat while the light curve of image $\mathrm{C}$ shows an increase in the first season (Fig. 3), the time delay between $\mathrm{C}$ and $\mathrm{B}$ (with $\mathrm{C}$ leading) must be larger than 560 days. Assuming $\mathrm{C}$ is leading, there is a minimum near $681 \pm 15$ days that corresponds to aligning the minimum observed in the first season for $\mathrm{C}$ with that observed in the last season for A/B. Due to the very different shapes of the light curves of images $A / B$ and $D$ there is no obvious solution over the whole observed time span. The only possibility would be to match the plateau in the third-year data of image $\mathrm{D}$ with the initial portions of the first-year data of images $\mathrm{A} / \mathrm{B}$, but there is no good candidate minimum in the goodness of fit. Therefore, we conclude that the time delay between A and D is larger than 800 days.

\section{MODELS AND INTERPRETATION}

We modeled the system using lensmodel (Keeton 2001) and the same component positions as were used by Kawanao \& Oguri (2006) and Inada et al. (2005). We fitted all five quasar images assuming astrometric minimum uncertainties of $0.003^{\prime \prime}$ and $100 \%$ flux uncertainties. We include the flux ratios very weakly because we only want to use them to enforce the image parities. We used the accurate but slow image plane fitting method, and the Hubble constant was fixed at $H_{0}=72 \mathrm{~km} \mathrm{~s}^{-1} \mathrm{Mpc}^{-1}$. The brightest cluster galaxy was modeled as an ellipsoidal de Vaucouleurs model with a major axis effective radius of $R_{e}=$ $4.8^{\prime \prime} \pm 0.5^{\prime \prime}$, an ellipticity of $0.18 \pm 0.02$, and a major axis position angle of $-12.8^{\circ} \pm 5^{\circ}$ based on fits to the CASTLES project's Hubble Space Telescope (HST) NICMOS H-band image of the system. The cluster halo was modeled as an ellipsoidal Navarro-Frenk-White (NFW) model with a break radius of $r_{s}=40.0^{\prime \prime}$ based on the mass model for the X-ray emission by Ota et al. (2006). We assumed priors on the ellipticity of the halo of $0.18 \pm 0.05$, no prior on its major axis position angle, and a prior on the external shear of $\gamma=0.05 \pm 0.05$. We also imposed hard limits on the ellipticity and position angle of the central galaxy $\left(0.15\right.$ to 0.25 and $-22^{\circ}$ to $\left.-2^{\circ}\right)$, the galaxy position $\left( \pm 0.3^{\prime \prime}\right.$ in each coordinate), the ellipticity of the halo $(0.0$ to 0.5$)$, the position of the halo $\left( \pm 3.0^{\prime \prime}\right.$ relative to the central galaxy) and the shear $(0 \leq \gamma \leq 0.25)$.

We first ran a model sequence based on simply adding a halo to the central galaxy. We started by fitting a de Vaucouleurs model with no halo to get the mass scale needed for the central galaxy in the absence of a halo. Then we fitted a series of models with the mass of the central galaxy fixed to a fraction $0 \leq$ $f \leq 1$ of its value in the no halo model. We ran the series both with and without the putative fifth quasar image. In general, the results are poor. The best fits to the image positions are obtained for $f \simeq 0.1$. The time delays for these models strongly disagree with our measurement in the sense that the model A-B delays are too short $(\sim 15$ days for $f=0.1)$. Producing a longer delay requires a model with a lower surface density near the images, since the time delays of these simple models are roughly proportional to $1-\langle\kappa\rangle$, where $\langle\kappa\rangle$ is the mean surface density in the annulus between the images (see Kochanek 2002). However, the models with $f \simeq 0.7$ and a low surface density that fit the delay correctly, fit the images poorly and have ellipticities for both the galaxy and the halo that are driven to their maximum permitted values because a side effect of lowering the surface density is to increase the required ellipticity (see Kochanek 2006b). That these simple models fit our delay measurement poorly is not surprising since the published results based on these simple model classes ${ }^{11}$ never produced a delay as long as our measured value.

The fundamental problem with this model, and all the preceding models of Oguri et al. (2004), Williams \& Saha (2004), and Kawano \& Oguri (2006), is that they neglect or poorly represent the substructure in the potential due to the presence of the other cluster galaxies. Many of these galaxies have deflection scales that are enormous compared to the astrometric uncertainties in the image positions, and as we painfully learned over

11 The nonparametric models of Williams \& Saha (2004) are roughly comparable in their overall structures. We note in passing that the discrimination between radial mass profiles observed in the nonparametric models is purely an artifact of the priors used in the analysis - there is a mathematical degeneracy that makes it impossible to use the positions of images A-D to determine the radial mass profile without the further assumptions supplied by the priors (see Kochanek 2006b). In our case, adding image E partly breaks the degeneracy. 


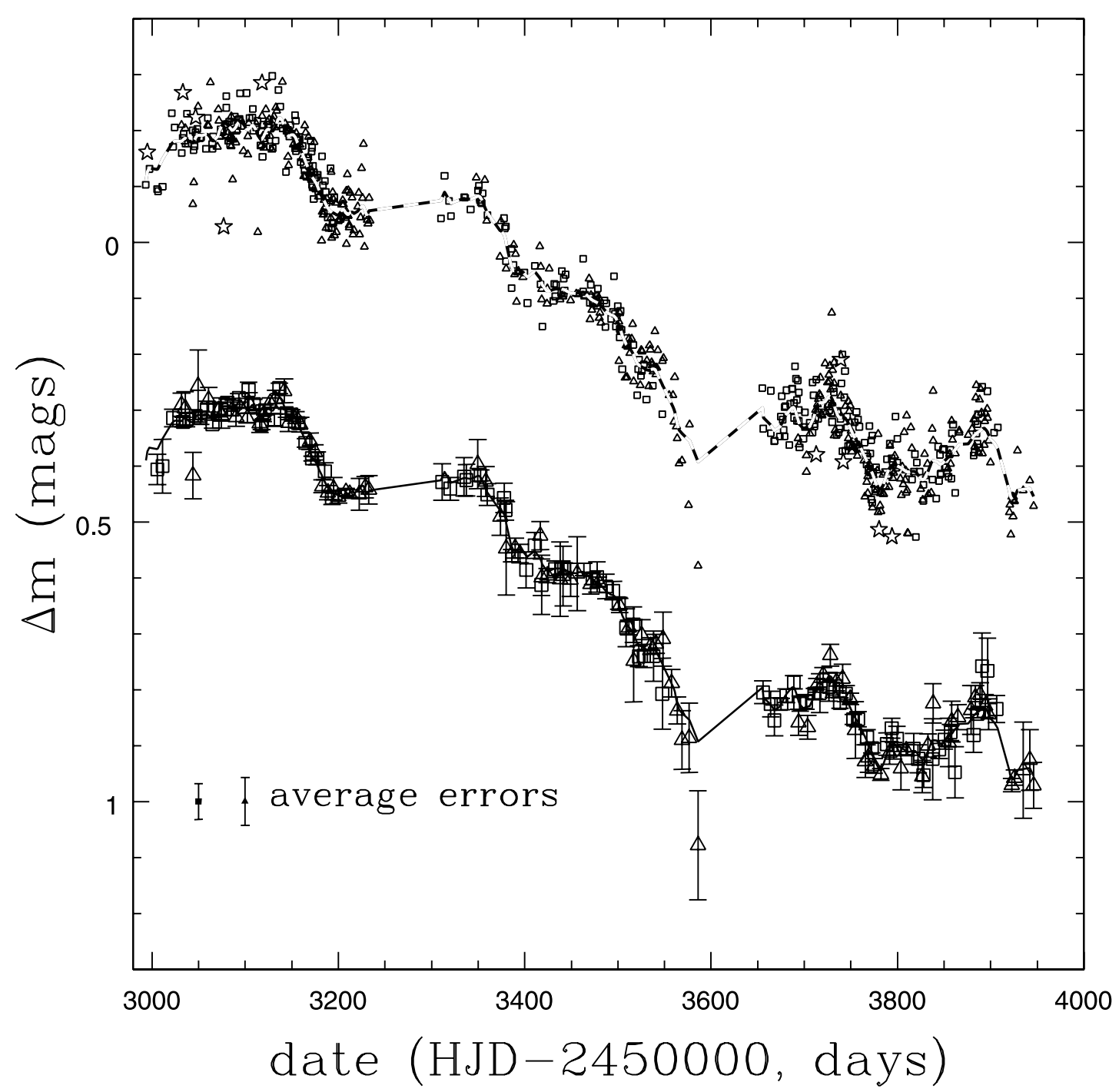

FIG. 8. - Estimated source light curve. The top curve presents the overlapping A (squares) and B (triangles) light curves after shifting by the time delay and subtracting the estimated microlensing variability. The curve is the best fit $N_{\mathrm{src}}=60$ source light curve model. There are three independent fits, one for each season, connected by a line through the seasonal gaps. The stars are the points that were masked in the time delay analysis. To minimize the confusion we are showing only the data with uncertainties smaller than $0.1 \mathrm{mag}$ and include examples of their mean error bars in the lower left corner. The bottom curve shows the same data binned in 1 week intervals, shifted downward by $0.5 \mathrm{mag}$ with error bars for the bins.

20 years of modeling Q0957+561, astrometric uncertainties can be imposed to no greater accuracy than the deflection scales of the most massive neglected components of the mass distribution (see Keeton et al. 2000; Kochanek 2006b). The neglect of the galaxies also affects the Shapiro delays, but the indirect effect on the delays through the deflections should dominate because the extra spatial derivative increases the importance of lowmass components relative to high mass components. In short, the model sequence we just considered, as well as all published models of this system, was virtually guaranteed to be quantitatively incorrect.

At a minimum, the model needs to include galaxies whose deflections cannot be trivially mimicked by rescaling the mass of the central galaxy and modifying the external shear. We used SExtractor (Bertin \& Arnouts 1996) to determine the positions and fluxes of the galaxies in the CASTLES HST ACS I-band image of the cluster. We assumed the galaxies had critical radii that scaled with the square root of their flux (i.e., SIS models obeying a Tully-Fisher relation) and added the 11 most important galaxies within $20.0^{\prime \prime}$ of the main lens galaxy as circular
pseudo-Jaffe models $\left[\rho \propto r^{-2}\left(r^{2}+a^{2}\right)^{-1}\right]$ with a break radius of $a \equiv 1.0^{\prime \prime}$. This truncates the galaxy halos as we would expect for galaxies in a cluster and the particular choice for the scale is relatively unimportant, since we are adjusting their mass normalizations. We required that they have mass scales (Einstein radii) in the range $0.05^{\prime \prime} \leq b \leq 2.0^{\prime \prime}$ and kept their positions fixed (see Table 2). We did not attempt to force a correlation between flux and Einstein radius as the scatter in the relation is fairly large (Rusin et al. 2003). Figure 10 shows the positions of these galaxies relative to the image positions and the cluster center. We then ran the same sequence of models for the central galaxy and halo. These models have no difficulty fitting both the A/B time delay and the $\mathrm{A}-\mathrm{D}$ image positions with reasonable parameters and a dark-matter-dominated cluster model $(f \simeq 0.1)$. Given the sensitivity of the A/B delay to substructure, it is probably premature to use the time delays as a strong constraint on the structure of the cluster. Reasonable models predict $\mathrm{B} / \mathrm{C}$ delays of order 450 to 1000 days, suggesting that the roughly 680 day solution in $\S 3.4$ may well be correct, and that the A/D delays are of order 5-7 yr. These longer delays should be much less sensitive 


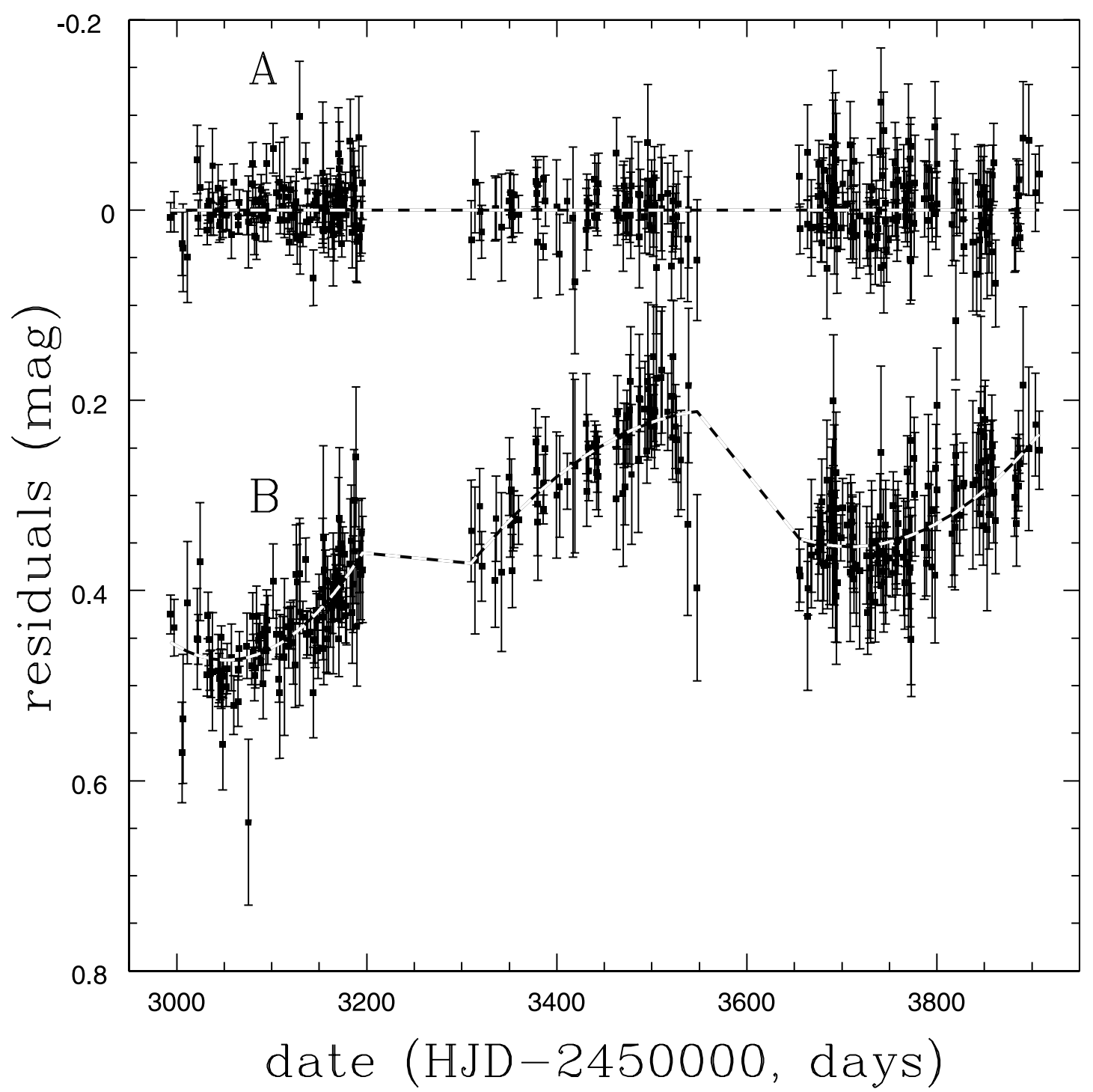

FIG. 9.- Inferred microlensing variability. We assigned the microlensing variability to image B, leaving the model for image A as a constant. This is an arbitrary choice that has no effect on the time delay - all we can really measure is the differential microlensing between the two images. The curves show the constant model used for A, the quadratic model $\left(N_{\mu}=3\right)$ for B and only the data with uncertainties smaller than 0.1 mag. There are three independent fits, one for each season, connected by a line through the seasonal gaps.

to the perturbations from galaxies and will provide a better basis for studying the cluster.

Figure 10 shows the critical line structure of an illustrative model. Since the best models lead to B/C delays reasonably close to the value of 681 days found by matching the minimum in the first season for $\mathrm{C}$ with those in the last season for $\mathrm{A} / \mathrm{B}$, we added it as a constraint ( $681 \pm 15$ days). The model fits the $\mathrm{A}-\mathrm{D}$ image positions very well $\left(\Delta \chi^{2} \simeq 1.3\right)$, and E less well $\left(\Delta \chi^{2} \simeq 13\right)$ due a $0.03^{\prime \prime}$ misfit to its right ascension. The problem in fitting $\mathrm{E}$ also leads to problems in the position of the main lens galaxy, which is driven to be $0.1^{\prime \prime}$ west $\left(\Delta \chi^{2}=12\right)$ of its measured position. The model fits the $\mathrm{A} / \mathrm{B}$ time delay perfectly, but it is $2 \sigma$ off (651 rather than 681 days) on the B/C delay. The very weak restrictions on the flux ratios make no contribution to the total $\chi^{2}=68$. In fact, the biggest contribution of $\Delta \chi^{2}=24$ to the total comes from the restriction of $0.18 \pm 0.05$ we placed on the ellipticity of the cluster. Thus, the problems in our fit are driven by image $\mathrm{E}$ and our restrictions on the shape of the cluster halo. In this model, the $\mathrm{D}$ image time delay relative to $\mathrm{A}$ and $\mathrm{B}$ is approximately $5.7 \mathrm{yr}$ and the central galaxy has $10 \%$ of the mass it would require in the absence of the dark matter halo. The
TABLE 2

A Representative Model

\begin{tabular}{|c|c|c|c|c|c|c|}
\hline Component & $\begin{array}{c}\text { Scale } \\
(\operatorname{arcsec})\end{array}$ & $\begin{array}{c}\text {-R.A. } \\
(\operatorname{arcsec})\end{array}$ & $\begin{array}{c}\text { Decl. } \\
(\operatorname{arcsec})\end{array}$ & $e$ & $\begin{array}{l}\text { P.A. } \\
\text { (deg) }\end{array}$ & $\begin{array}{c}\text { Size } \\
(\operatorname{arcsec})\end{array}$ \\
\hline deVauc........... & 2.800 & 7.214 & 4.390 & 0.219 & -18.68 & 4.717 \\
\hline 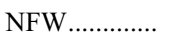 & 0.272 & 6.593 & 5.161 & 0.425 & -5.02 & $\equiv 40.0$ \\
\hline p-jaffe ............ & 0.483 & 9.231 & 2.503 & $\equiv 0$ & $\ldots$ & $\equiv 1.0$ \\
\hline p-jaffe ............ & 0.884 & 12.190 & 3.691 & $\equiv 0$ & $\ldots$ & $\equiv 1.0$ \\
\hline p-jaffe ............ & 1.381 & 2.117 & 11.850 & $\equiv 0$ & $\ldots$ & $\equiv 1.0$ \\
\hline p-jaffe ............... & 0.736 & 2.766 & -0.177 & $\equiv 0$ & $\ldots$ & $\equiv 1.0$ \\
\hline p-jaffe ............ & 0.050 & 2.757 & 14.120 & $\equiv 0$ & $\ldots$ & $\equiv 1.0$ \\
\hline p-jaffe .............. & 0.073 & 14.780 & -5.466 & $\equiv 0$ & $\ldots$ & $\equiv 1.0$ \\
\hline p-jaffe ............. & 0.071 & 4.397 & -0.617 & $\equiv 0$ & $\ldots$ & $\equiv 1.0$ \\
\hline p-jaffe ............. & 1.436 & 9.458 & 18.620 & $\equiv 0$ & $\ldots$ & $\equiv 1.0$ \\
\hline p-jaffe ............. & 0.211 & -1.365 & 0.489 & $\equiv 0$ & $\ldots$ & $\equiv 1.0$ \\
\hline p-jaffe ............. & 1.998 & 12.160 & 13.530 & $\equiv 0$ & $\ldots$ & $\equiv 1.0$ \\
\hline p-jaffe .............. & 0.375 & 6.527 & 11.340 & $\equiv 0$ & $\ldots$ & $\equiv 1.0$ \\
\hline
\end{tabular}

Notes.-The model definitions are as in Keeton (2001) and the positions are relative to image A. The sizes are the effective radius for the De Vaucouleurs model and the break radius for the NFW and pseudo-Jaffe models. The shear parameter is $\gamma=0.175$ with orientation $-80.70^{\circ}$. 


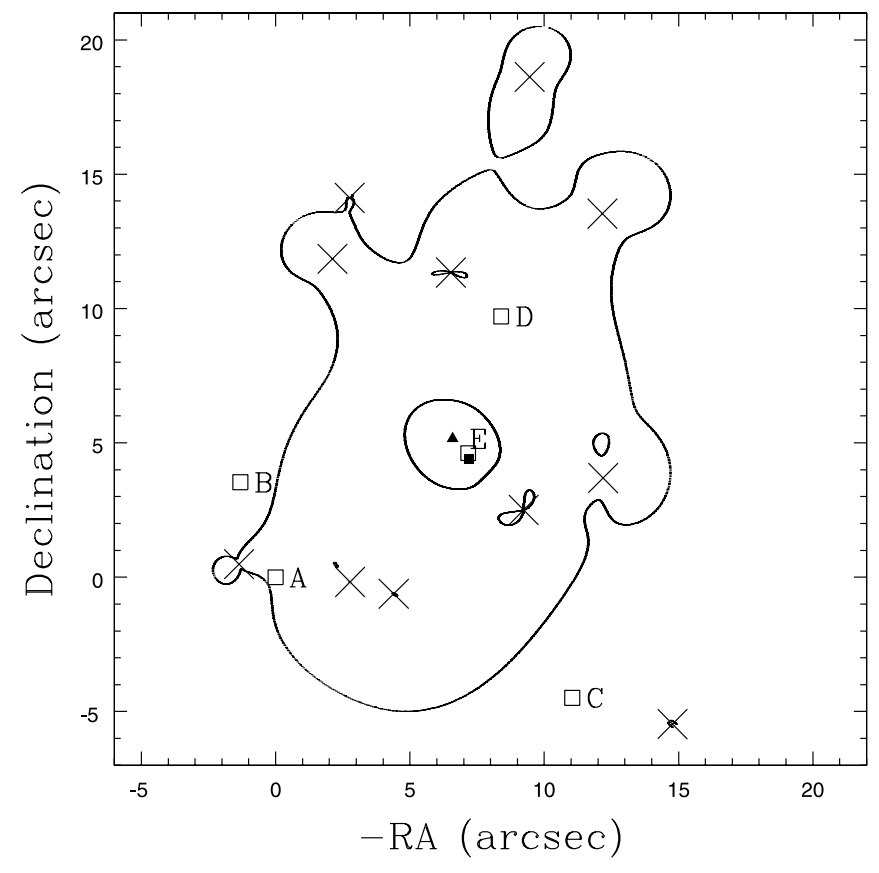

FIG. 10.-Lens model generally consistent with the data. The open squares show the positions of the five lensed images A-E. The solid lines show the critical curves of the lens model, whose considerable, but realistic, complexity is due to the presence of the main lens galaxy (filled square), an offset halo (filled triangle), and 11 smaller galaxies (large crosses). The model fits the time delays and the image positions nearly perfectly.

Einstein radii of the smaller galaxies range from $0.05^{\prime \prime}$ to $2.0^{\prime \prime}$, which are plausible mass scales. The offset between the main lens galaxy and the dark matter halo found in earlier models still seems to be required. In any case, it is not our present intent to conduct a full model survey, but to emphasize the need for more realistic models.

Figure 10 illustrates the extend to which adding the member galaxies can modify the critical line structure of the lens even if the particular model has flaws.

\section{SUMMARY AND CONCLUSIONS}

We have measured the A/B time delay of SDSS J1004+4112 to be $(38.4 \pm 2.0)$ days $\left(\Delta \chi^{2}=4\right)$, which fixes the overall time ordering of the images to be C-B-A-D-E. While this is the time ordering predicted in published models (Oguri et al. 2004; Williams \& Saha 2004; Kawano \& Oguri 2006) it is significantly longer than the delays predicted by these models. The cause of the discrepancy is that the previously published models overly simplified the mass distribution by neglecting the deflections generated by the cluster member galaxies.

Models including the 11 most important galaxies can simultaneously fit the A-E image positions and the measured A/B time delay with reasonable parameter values. Modelers of this system need to remember the lesson of Q0957+561: model constraints that are applied more tightly than the deflection scale of the most massive, neglected components of the lens lead to incorrect results (Keeton et al. 2000). We note that Sharon et al. (2005) also needed to include some of the member galaxies in order to model the higher redshift lensed arcs, but made no predictions for the time delay. Including galaxies in the mass model is necessary, but it may not be sufficient, as massive substructures in the cluster may not be limited to the visible galaxies. Deeper $\mathrm{X}$-ray observations may be able to search for high-mass sub- structures in the cluster halo, as there is some disagreement on the regularity of the X-ray emission between the Chandra study by Ota et al. (2006) and the XMM-Newton study of Lamer et al. (2006).

Fortunately, the A/B delay should be the most sensitive of the delays to the effects of cluster galaxies because it is a merging image pair. The longer delays for the $\mathrm{C}$ and $\mathrm{D}$ images relative to $A$ and B should be less affected by substructure, so their measurement should provide constraints on the cluster halo properties that are less sensitive to the member galaxies. At present we cannot claim a measurement of these longer delays. A lower bound on the delay $\Delta t_{\mathrm{AD}}>800$ days is consistent with our models, which predict delays of 5-7 yr for this image pair. The shorter $\mathrm{C} / \mathrm{B}$ delay is at least $\Delta t_{\mathrm{CB}}>560$ days, but there is a possible delay of $\Delta t_{\mathrm{CB}} \simeq 680 \pm 15$ days that should be confirmed or rejected during the next observing seasons and is consistent with our models.

We have also clearly detected microlensing variability in the A/B images, with changes of order $0.15 \mathrm{mag}$ in the A/B flux ratio over the course of the three observing seasons. This result provides strong evidence that the differential changes in the A/B emission line profiles are also due to microlensing (Richards et al. 2004; Lamer et al. 2006; Gómez-Álvarez et al. 2006) rather than variable absorption in the source (Green 2006). The microlensing timescales in SDSS J1004+4112 should be relatively shorter than in most single galaxy lenses because the internal velocities of the cluster are about 3 times higher than those of a galaxy. While the flux ratio changes in the optical continuum are modest, we would expect to find significantly larger effects at shorter wavelengths, where the source size should be more compact. There is already some evidence for this from the X-ray flux ratios measured by Ota et al. (2006) and Lamer et al. (2006). A campaign to monitor this system in X-rays would both allow us to study the size of the X-ray emission region and provide the added data on the emission from the cluster needed to provide a precision comparison of the mass distributions estimated using $\mathrm{X}$-ray data and lens models. Such careful tests will be essential if measurements of the increase of the Einstein radius of the cluster with source redshift based on the surrounding multiply imaged arcs are to be used as a new test of the cosmological model as proposed by Soucail et al. (2004) and Sharon et al. (2005).

We thank the Smithsonian Astrophysical Observatory (SAO) and all their participating observers for the support of our observations. This work is based on observations obtained with the Apache Point Observatory $3.5 \mathrm{~m}$ telescope, which is owned and operated by the Astrophysical Research Consortium. We thank E. Turner for organizing the APO observations. This work is also based on observations obtained with the MDM $2.4 \mathrm{~m}$ Hiltner and $1.3 \mathrm{~m} \mathrm{McGraw}-H i l l$ telescopes, which are owned and operated by a consortium consisting of Columbia University, Dartmouth College, the University of Michigan, the Ohio State University, and Ohio University. We would like to thank J. Halpern, J. Patterson, and S. Tuttle of Columbia University, D. Depoy, J. Eastman, S. Frank, J. Marshall, J. Prieto, K. Stanek, and D. Terndrup of OSU, and J. R. Thorstensen of Dartmouth College for their observations of this system. Observations for this project at Wise Observatory were supported by grants from the German-Israeli Foundation for Research and Development and the Israel Science Foundation. The WIYN Observatory is owned and operated by the WIYN Consortium, which consists of the University of Wisconsin, Indiana University, Yale University, 
and the National Optical Astronomy Observatory (NOAO). This work is based in part on observations made with the NASA/ESA Hubble Space Telescope as part of programs HST-GO-9744, HST-GO-10509, and HST-GO-10716 of the Space Telescope Science Institute, which is operated by the Association of Univer- sities for Research in Astronomy (AURA), Inc., under NASA contract NAS5-26555. We also acknowledge support by the European Community's Sixth Framework Marie Curie Research Training Network Programme, contract MRTN-CT-2004-505183 "ANGLES."
Alard, C., \& Lupton, R. H. 1998, ApJ, 503, 325

Bertin, E., \& Arnouts, S. 1996, A\&AS, 117, 393

Dalal, N., \& Keeton, C. R. 2003, preprint (astro-ph/0312072)

Gil-Merino, R., Wisotzki, L., \& Wambsganss, J. 2002, A\&A, 381, 428

Gómez-Álvarez, P., Mediavilla, E., Muñoz, J. A., Arribas, S., Sánchez, S. F.,

Oscoz, A., Prada, F., \& Serra-Ricart, M. 2006, ApJ, 645, L5

Green, P. J. 2006, ApJ, 644, 733

Inada, N., et al. 2003, Nature, 426, 810 2005, PASJ, 57, L7

Kawano, Y., \& Oguri, M. 2006, PASJ, 58, 271

Keeton, C. R. 2001, preprint (astro-ph/0102340)

Keeton, C. R., et al. 2000, ApJ, 542, 74

Kochanek, C. S. 2002, ApJ, 578, 25

Kochanek, C. S., Morgan, N. D., Falco, E. E., McLeod, B. A., Winn, J. N., Dembicky, J., \& Ketzeback, B. 2006a, ApJ, 640, 47

Kochanek, C. S., Schneider, P., \& Wambsganss, J. 2006b, Gravitational Lensing: Strong, Weak, and Micro, ed. G. Meylan, P. Jetzer, \& P. North (Berlin: Springer)

\section{REFERENCES}

Lamer, G., Schwope, A., Wisotzki, L., \& Christensen, L. 2006, A\&A, 454, 493 Morgan, C. W., Byard, P. L., Depoy, D. L., Derwent, M., Kochanek, C. S., Marshal, J. L., O’Brien, T. P., \& Pogge, R. W. 2005, AJ, 129, 2504 Oguri, M., et al. 2004, ApJ, 605, 78

Ota, N., et al. 2006, ApJ, 647, 215

Pelt, J., Hoff, W., Kayser, R., Refsdal, S., \& Schramm, T. 1994, A\&A, 286, 775 Pelt, J., Kayser, R., Refsdal, S., \& Schramm, T. 1996, A\&A, 305, 97 Press, W. H., Rybicki, G. H., \& Hewitt, J. N. 1992, ApJ, 385, 416

Richards, G. T., et al. 2004, ApJ, 610, 679

Rusin, D., et al. 2003, ApJ, 587, 143

Sand, D. J., Treu, T., Smith, G. P., \& Ellis, R. S. 2004, ApJ, 604, 88 Sharon, K., et al. 2005, ApJ, 629, L73

Soucail, G., Kneib, J.-P., \& Golse, G. 2004, A\&A, 417, L33

Vanden Berk, D. E., et al. 2004, ApJ, 601, 692

Wambsganss, J. 2003, Nature, 426, 781

Williams, L. L. R., \& Saha, P. 2004, AJ, 128, 2631 